Visual analysis in social representations of happiness research Abstract

This study of social representations of happiness includes visual analysis of photographs as one of the instruments of qualitative methodology. Lately the term 'visual culture' has been frequently used as one of the characteristics of the modern world. Photography as an object of qualitative analysis helps us approach the decoding of complicated social-psychological phenomena. The object of this research is social representations of happiness. The subject is the contents of social representations of happiness. The goal of the study is to describe the contents of social representations of happiness, using the collected verbal and non-verbal data. The carriers of social representations of happiness in our work will be the students of Moscow State University. We have collected and received collections of photos and commentary to them from seven individuals between the ages of 21 and 24. Furthermore, these photo collections were analysed. Analysis included four stages: hermeneutic, semiotic, structural and discursive. The appliance of the visual method, aimed to study substantive and non-static relationships, assisted in discovering the core and the periphery of social representations of happiness. This way we fixate the significance of the iconic component when researching social occurrences in the digital world.

\section{Stages of research}

I) The group was offered to create a photo collection on the topic of happiness, which would comprise of five to seven photographs within the timeframe of a month;

2) the respondents gave titles to their photographs and provided arguments regarding the inclusion of the photographs in their collection;

3) these photo collections underwent primary analysis, and then were analysed. It included four types of analysis: hermeneutic, semiotic, structural and discursive.
Mel'nikova Ol'ga, Izbasarova Sofia Lomonosov Moscow State University, Department of social psychology

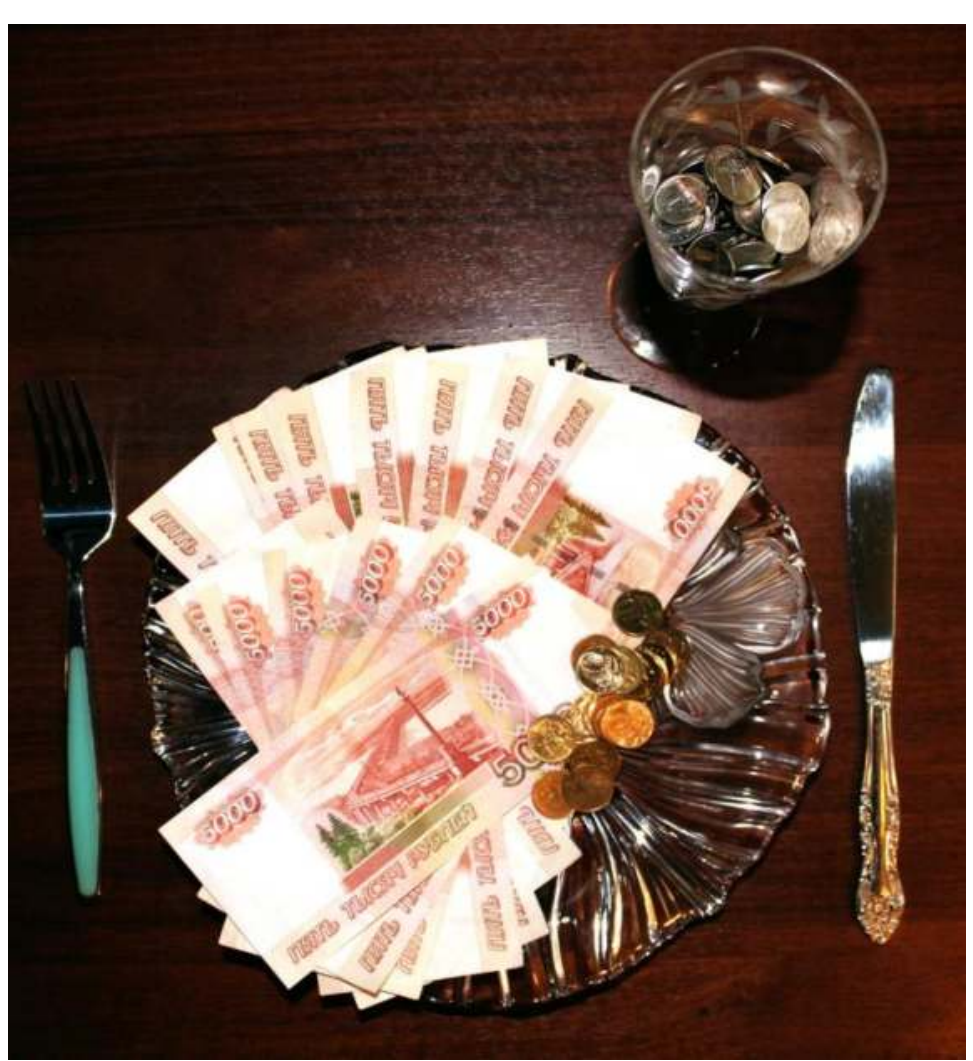

The carriers of social representations of happiness in our work will be the students of Moscow State University. We have collected and received collections of photos and commentary to them from seven individuals between the ages of 21 and 24. The quantity of students was determined due to the specifics of qualitative research, that entails descriptive saturation of data, which is possible with the number of subjects being between 6 and 8 individuals.

\title{
Results
}

Social represt In terms of factors that affect happiness, the key ones involve significant to the participants figures: family, friends, a partner, children or pets. This content can be viewed as the core of social representation of happiness, as in one way or another it is reflected in every photo story, and in some of them it is even used more than once. At the periphery of social representation of happiness is the category of travel, which has two sides: aesthetic pleasure and self-development. Quite often this pastime is spent with a significant person. Another component is spiritual development as self-development and meeting new people of interest.

In every collections of photos, on the majority of photographs, whatever area they illustrate, the characteristics of prestige are visible. This concerns even friends and significant others, that are presented on the photographs as equals in terms of status and their financial state. Wealth acts as a 'foundation' that is necessary for happiness, yet without other elements money does not bring happiness.

the focus is on the analysis of the photographer's personality: what they wanted to communicate (what knowledge), what preconceptions and stereotypes they believe in what personal experience is involved, what emotions are channelled. On the stage of semiotic interpretation, the denotation and connotation of representations were counterposed. Denotation is everything that the image vividly demonstrates and what the sign correlates to. Connotation represents more complex associations, thoughts, feelings, that are awaken by the image (the sign). In terms of structural interpretation, we used the INIS chart to recognize the declared social relationships, that include the following characteristic for analysis:

I) Forms of interactions in the society (I interactions);

2) social rules that describe the desired way or style of behaviour ( $\mathrm{N}$ - norms);

3) ideas that are spread in the society, their views and prejudices (I - ideas);

4) the possibility of accessing valuable amenities, e.g. wealth, prestige, education, health (S chance).

The discursive interpretation implicates the analysis of the audience that the photo is targeted towards.

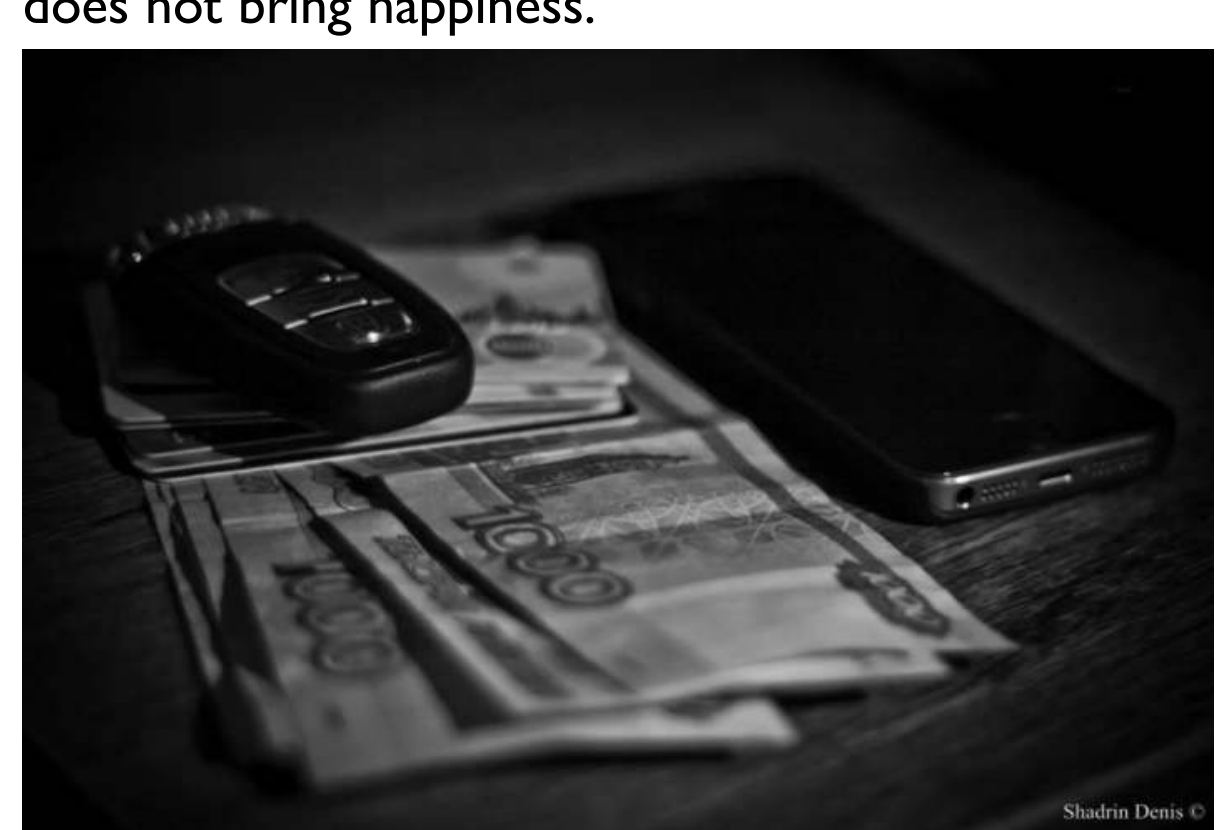

Our further scientific work

During the last several years, while considering the qualitative research, a lot of attention was paid not to the criteria of the evaluation of results of the study but to the exact strategies of 'internal' verification of the research process to eliminate the potential dangers to validity. A special place is allocated to triangulation. Triangulation can also be called the design of mixed research, which creates a major trend in modern social sciences and psychology. Our further scientific work will be dedicated to the development of this research design with the use of triangulation as an additional strategy of validation. 